\title{
BEATS FÜR DIE HEIMATFRONT: »LET'S GET LOUD «. Die Kulturindustrie MOBILISIERT FÜR DEN KRIEG
}

\author{
Susann Witt-Stahl
}

Als die Popstars sich nach dem 11. September 2001 reihenweise mit dem Feldzug der USA gegen Afghanistan aktiv solidarisierten und sich zumindest für die Mobilisierung der Heimatfront zur Verfügung stellten, zeigte sich die kritische Pop-Fachwelt erstaunt über den militanten Westliche-WeltPatriotismus, vor allem aber alarmiert durch den unverhohlen performativen Bellizismus der Popmusik-Szene - gemeint ist hier der Mainstream. Bis zum 11. September genoss die Popmusik den Ruf des Friedensbotschafters, der im Stande war, Massenproteste gegen das »Töten in Haufen« - wie Elias Canetti in Masse und Macht 1960 den Krieg nannte - wenn auch nicht auszulösen, so doch mindestens ästhetisierend zu unterstützen. Spätestens seit Vietnam und Woodstock wurde Pop als Befreiungskultur, als Sprache der Schwachen und Verlierer oder gar als Refugium für das widerständige Subjekt gewürdigt. Stellt sich die Frage: Hat Pop nach dem 11. September die Seiten gewechselt - von einer Massenkultur des Friedens zur Kultur der Macht und Gewalt? Oder ist das legendäre Engagement der Popmusik für den Frieden eine Lüge aus dem schier unerschöpflichen Massenbetrugsrepertoire der Kulturindustrie? Mehr noch: Sind Pop und Krieg nicht sogar genuin miteinander verbunden?

Wenn bisher von Mobilisierung von Pop für den Krieg die Rede war, dann assoziierte man die Propagandaschlager der Weltkriege, Frontunterhaltung und Soldatensender. Das ist natürlich - die Monate nach dem 11. September, eine Zeit totaler Hysterie, bilden eine Ausnahme - weitgehend Vergangenheit. Die Suche nach Popstars, die als Frontunterhalter auftreten, und nach Musiktiteln, in denen beispielsweise der Irakkrieg der USA propagiert, zumindest gerechtfertigt wird, endet nahezu ergebnislos: Außer »Let's Roll« von dem strammen Republikaner Neil Young - übrigens einem Protagonisten der Protestbewegung gegen den Vietnamkrieg -, Charlie Daniels' »This Ain't No Rag It's A Flag« oder Darryl Worleys »Have You Forgotten« (»how it felt that day to see your homeland under fire and her people blown 
away?«) - aus der Sparte Country ist man von jeher patriotische bis chauvinistische Töne gewöhnt - können kaum Titel angeführt werden, die sich positiv auf den Krieg beziehen. Das ist kein neues Phänomen: Popmusik als Kriegspropaganda hat von jeher nur in seltenen Fällen dezidiert, manifest für den Krieg geworben. Aus dieser Tatsache lässt sich jedoch nicht ableiten, dass Popmusik in der Reklame für den Krieg keine Rolle mehr spielt.

Schauplatz ist irgendeine Militärbasis in Pakistan: "Let's get loud«, appelliert Jennifer Lopez an die tobende Meute und tänzelt im Combat-Oufit über die Bühne. Sie fordert die US-amerikanischen Soldaten zum rhythmischen Klatschen auf: "Life is meant to be big fun, / You're not hurting anyone $«$. Kriege machen Spaß, und Soldaten tun niemandem etwas zu Leide.

Superstars des Vulgär-Pop, wie Mariah Carey oder Geri Halliwell, setzten nach dem 11. September wieder auf Mobilisierung und ließen sich in den Armen von stiernackigen Elite-Fightern ablichten. Während bundesdeutsche Soldaten im Feldlager zu Prizren noch Machwerke wie »Es steht ein Haus im Kosovo « von dem Trucker- und Malocher-Barden Gunter Gabriel über sich ergehen lassen mussten, wurden ihre US-amerikanischen Kameraden von den Stars aller Sparten der Unterhaltungsindustrie regelrecht hofiert. Rapper Coolio, Hardrockerin Joan Jett, Reggae-Sänger Shaggy, Soul-Legende Ruth Pointer tourten im November 2001 von Stützpunkt zu Stützpunkt. Die australische Sängerin Kylie Minogue trat bei den britischen Soldaten in der Golfregion auf. Zur Jahreswende veranstaltete die Agentur der United Service Organizations (USO), gesponsert von Multis wie Coca Cola und Yahoo, zusammen mit dem Sender MTV das Mega-Spektakel For The Troops. Jennifer Lopez, Ja Rule und Kid Rock waren als Top-Acts angekündigt:

" I've always considered myself to be a pretty patriotic guy, I don't know if you've been living in a cave or not, $<$ Kid Rock said. >I feel that it's my part as an American [to participate]. If they needed me to pick up a gun, I'm happy to do that too, but I think this is my place. So right now, I'm ecstatic to go over and entertain the people who are fighting for my freedoms, because I do take advantage of my freedoms.< For his performance, Kid Rock said he's got a medley of classic American rock tunes planned. >I'm going to bring them a show that's going to peel their wig back, « he boasted. >Those guys are going to forget we're at war« (Vineyard 2001).

Zehn Tage nach der WTC-Tragödie konnte man schon erste MobilisierungsSignale von der Pop-Prominenz wahrnehmen. Während der A Tribute To Heroes-Gala am 21. September in New York boten die Troubadoure der »freien Welt « den bärtigen Terroristen musikalisch die Stirn und versicherten aufrichtigen Durchhaltewillen. Der von Paul McCartney einen Monat später zum von ihm selbst im Madison Square Garden organisierten Concert 
For New York City beigesteuerte Titel »Freedom«, den er eigentlich der "greatest democracy on earth « gewidmet hat, ist ein hervorragendes Beispiel für die integrative Kraft des Mainstream-Pop. Der Text lässt nämlich keine Außenseiter zu, bietet Identifikationsfläche gleichermaßen für Kriegsbefürworter und -gegner - kein unwichtiger Aspekt, bedenkt man, dass die Harmonisierung von Zwangsgemeinschaften, eine möglichst stabile Heimatfront, für die erfolgreiche Durchführung von Kriegen noch unabdingbar ist und die Kulturindustrie schließlich auch ökonomische Interessen verfolgt.

Deutlicher wurde die Kulturindustrie am 21. Oktober, einen Tag nach McCartneys Konzert, beim United We Stand-Konzert in Washington. Veranstalter und Clear Channel Entertainment-Vorstand Brian Becker versprach Präsident Bush offiziell die Unterstützung der Mitwirkenden für die Militärs, weil diese »are risking their lives for our safety« (zit. n. Jeckell 2001). Der Schauspieler Richard Gere, bekennender Buddhist, hatte es schwer. Mit seinem vorsichtig geäußerten Wunsch nach »Peace « handelte er sich deftige Flüche und Buhrufe von der revanchegeilen und unentwegt »USA! USA! « skandierenden Jubelmeute im Auditorium der Heroes-Gala ein. Die Rubrik »dona nobis pacem« war nach dem 11. September nahezu getilgt aus dem Programm der Kulturindustrie. Die Platzierung einer HipHop-Version von Country Joe McDonalds »I-Feel-Like-I'm-Fixin'-To-Die Rag« in den höheren Rängen der Charts war nicht zu erwarten und vor allem nicht erwünscht. Denn die westliche Welt kämpfte gegen das reine Böse. Frontunterhaltung, nach Vietnam verpönt und bestenfalls von zweitklassigen Country-Bands oder ausgemusterten Models zum Einspielen ihres Gnadenbrots genutzt, erstrahlte wieder im Glanz vergangener Tage, als noch Marlene und Marilyn »for the boys« sangen. Pop's coming home, in den Schoß des Krieges.

Pop wäre ohne die enge Verflechtung von Kommunikationstechnik und Kriegsführung, ohne die gigantischen Technisierungsschübe des »Vaters aller Dinge « nicht möglich gewesen. Pop ist Abfallprodukt und Waffe des Krieges zugleich. Ende des 19. Jahrhunderts, als Pop anfing, Pop zu sein - das heißt als industriell gefertigte und standardisierte Ware erhältlich -, war Pop mit der Militärmusik noch vielfältig verwoben. Militärmusikkultur und zivile Unterhaltung erwiesen sich zumindest als wechselseitig durchlässig. Der Marsch war aus seinem militärisch-funktionalen Bereich herausgetreten, zur Darbietungs- und Unterhaltungsmusik gereift und zum Crossover mit zivilen Genres - von der Oper bis zum Gassenhauer - angetreten. Militärmusiktrainer wie Geoffrey O'Hara schrieben Kriegshits, in denen alle möglichen Sujets verarbeiten wurden, nur nicht der Krieg. Es waren dann auch Militärbands, die die Vorboten des Jazz nach Europa brachten und Musiken jeglicher Provenienz ad usum populi servierten. Im Gegenzug verbanden zivile 
Komponisten wie der deutsche Paul Lincke das genuin europäische Genre Marsch mit afro-amerikanischen Synkopen, pentatonischen Wendungen und Polyrhythmik. Die Unterhaltungsindustrie transportierte den Starkult des gleichzeitig heroischen und bonvivanten Leutnants als »Offizier und Gentleman«, der zum Vorbild der männlichen und zum Objekt erotischer Fantasien der weiblichen Pubertierenden wachsen sollte. Ob Marsch oder Operette, Couplet oder Schlager, Cake Walk oder Ragtime: Die Stilisierung, Banalisierung und vor allem Verkitschung des Krieges war unaufhaltsam. Krieg sollte so lustig werden wie noch nie.

Das änderte sich auch während des Zweiten Weltkriegs nicht, mit einem wichtigen Unterschied: Pop, mit all seinen Sparten, hatte die Führung in den Propagandaschlachten übernommen. Der Militärmarsch spielte als Genre eine nebengeordnete Rolle, militärische Zeichenqualitäten jedoch waren in der Popmusik in großer Fülle vorhanden. Der Swing, die populärste U-Musik des Zweiten Weltkriegs, enthält viele Stilelemente der Militärmusik: beispielsweise die Dominanz der Bläser, das Schlagzeug als Präzisionsmaschine oder die disziplinierte Trennung von melody section und rhythm section. Der feste Tritt der Nagelstiefel war dem Offbeat der großen Show-Orchester von Teddy Powell oder Teddy Stauffer gewichen. Der Swing mit seinen sensualistischen Reizen, implantierten Gimmicks und seinem hektischen Drive ließ den Krieg als spaßige touristische Attraktion erscheinen. Bing Crosby und die Andrew Sisters versprachen eine »Hot Time In The Town Of Berlin«. In anderen Sparten sah es nicht anders aus. Schnulzen-Sänger Little Jack Little wünschte sich: »|'ve Always Wanted To Dance In Berlin« oder Country-Swinger Ozzie Waters träumte von einem »Rodeo Down In Tokyo«. Der berühmteste Kriegsheld trug zwar Uniform, war aber kein Soldat, sondern Donald Duck. Er bekämpfte seinen Kontrahenten Adolf Hitler nicht nur in Comic-Heften und -Filmen, sondern auch in überaus albernen Pop-Songs wie beispielsweise in Johnny Bonds »Der Führer's Face« von 1942.

Im nationalsozialistischen Deutschland dominierten nicht die berüchtigten Kampflieder von Hans Baumann oder Heinrich Spitta den Kriegsalltag, sondern vorwiegend Schlager, die hohe Schule der Tendenzlosigkeit und Anpassung. Insgesamt war in deutschen Schlagern ein - wie Lothar Prox (1979: 76) es nennt - »zupackender Gestus « einer Party-Gute-Laune-Musik verbreitet. Durch diesen Gestus (»Wir machen Musik, da geht euch der Hut hoch / Wir machen Musik, da geht euch der Bart $a b \ll$ ) wurde Spontaneität suggeriert - nicht kulturelle Arbeit, sondern des Menschen Naturzustand, worin er ungespalten mit Geist und Sinnen aufgeht und einen göttergleichen, verwandelten Status erreicht: »Mit Musik ist ja das ganze Leben 
nur noch halb so schwer «, und »Willst du einmal glücklich sein, dann hilft nur sie allein« (zit. n. ebd.). Die Heerschauen fanden nicht mehr auf dem Zeppelinfeld, sondern auf den Showtreppen der Revuefilme statt. Diese fungierten als Dressurschau der formierten Kriegsmasse:

»Wenn die Girls in >Wir tanzen um die Welt $<$ in triumphaler Siegespose allabendlich im Stechschritt die Treppe heruntermarschieren, treten sie in lackierten Stiefeln den noch unsichtbaren Aufstand nieder. Das ist Tanz im Dienste; die einen reiten für Deutschland, die anderen tanzen und exerzieren im Bereich der Unterhaltung eine Form vor, die ihren Ausdruck im Krieg findet« (Witte 1979: 8).

Das Bedürfnis nach vitaler, flotter, swingender Tanzmusik war auch im NaziDeutschland groß. Die Musikfilme wurden entsprechend aufgelockert. Sogar im »Wunschkonzert für die Wehrmacht« tauchten ab und zu gemäßigte Jazz-Titel auf.

Die Kriegsprodukte der Kulturindustrie des Zweiten Weltkriegs kennzeichnen sich jedoch nicht ausschließlich durch hysterische Tanzwut und trotzige Fröhlichkeit, sondern auch durch sehnsuchtsvolle Weltflüchtigkeit, Sentimentalität und Schicksalsergebenheit. Die Beliebtheit des WeltkriegHits »Lili Marleen«, über alle Fronten hinaus, ist auf die vielen semantischen Bezüge zum Soldatenalltag, besonders aber auf seine starke Abschiedsmetaphorik zurückzuführen. Ähnliches gilt für das Chanson "J'attendrai«, das in Deutschland unter dem Titel »Komm zurück! « erschienen war - die Nazis spielten es gern zu den Hinrichtungen von Häftlingen, die Fluchtversuche aus Konzentrationslagern unternommen hatten (Kuna 1993: 33).

$\mathrm{Ob}$ als scheinbar tendenzlose Begleitmusik, Ornamentierung und Kompensator des Kriegsalltags oder als Propagandawaffe: Theodor W. Adorno, der die Kulturindustrie bezichtigte, die Musik zum »Naturschutzpark der Irrationalität« verkommen lassen zu haben, hatte Recht mit seiner Behauptung, dass Pop eigentlich nur in zwei Typen vorkomme: Rhythmische Musik und sentimentale Musik (vgl. Adorno 1941: 40ff.). Zu diesen Typen, so der Musiksoziologe, gebe es korrespondierende Verhaltensweisen: Eine, die sich dem Rhythmus unterwerfe (»slave to the rhythm«) und dem autoritären Kollektivismus der Ganzheitsmaschine unterordne, und eine weitere, die das verwehrte, unerfüllte Glück realisiere und in der Musik die Lizenz zum Weinen finde. Adornos Fazit lautet: »One who weeps does not resist any more than one who marches « (ebd.: 42).

Pop fand nicht zu einer Sprache des Widerstands gegen den Krieg, auch nicht zu einer des Leidens und Schmerzes. Nicht einmal als Mobilisierungsmusik klagte er die unmenschlichen Verbrechen des Feindes an, sondern 
beschränkte sich auf ein Banalisieren oder maximal auf ein irrationales Perhorreszieren.

Mit den immer gleichen Mitteln lässt Pop das Irrationale triumphieren, vor allem durch eine rauschhaft-sinnliche Rhetorik, die ein glückliches Jetzt-Erlebnis verspricht. Treffen Pop und Krieg zusammen, erscheint das ästhetische Leitmotiv dieser massenkulturellen Ereignisse in bemerkenswerter Dichte. Die Intensität des Augenblicks erfährt eine hedonistische Huldigung. Sein exzessives Erleben verspricht die Befreiung von dem lästigen Regelwerk der Vernunft und Fluchthilfe aus der kulturellen Enge, den Fesseln der Moral und ihren kategorischen Imperativen. Der Rock'n'Roll sollte in seinen vielfältigen Erscheinungsweisen den Beweis dafür antreten.

In seiner Schrift Feuer und Blut von 1929 entwarf Ernst Jünger bereits das Drehbuch für Coppolas Apokalypse Now, diesem obszönen Thriller über die Faszination des Schreckens, der später zu einem ästhetischen Leitmotiv von Metal-Bands wuchern sollte. Bei Jünger heißt es:

»Und laßt das Bild der großen Schlacht aus dem Rausch aufschießen wie eine blutrote Blume, mit goldenen Feuerstreifen geflammt. Das ist ein Kunstwerk, wie es Männern Freude macht. Hier, wo die Leiden dunkler, aber auch die Lüste brennender und wilder sind, während des Tanzes über dem Abgrunde, lernt man das Blut schätzen und die dürftigen Geister verachten, wenn man der Feuerprobe gewachsen ist. Und das ist auch ein Auf- und Untergang. Aber vorher wollen wir ein Fest aus unserem Untergange machen, ein Fest, zu dem das Geschütz der ganzen Welt einen brüllenden, noch niemals gehörten Salut schießen soll« (Jünger 1929: 28f.).

Die Regression auf Trieb und Rausch, das ozeanische Gefühl bis zur bedingungslosen Hingabe an den dionysischen Exzess sollte dann den »ersten Rock'n'Roll-Krieg (Herr 1979) in Vietnam dominieren.

Die meisten Produkte der Kulturindustrie dieser Zeit thematisierten das intensive Lebensgefühl einer Jugend, die ihre gähnende Langeweile in rauschhafte oder berauschte Fantasien umgewandelt hatte. Wie John Hellman (1986) in seiner Abhandlung American Myth and the Legacy of Vietnam ausführlich darlegt, gehörte dazu auch der aufrichtige Glaube an eine neue Spielart des »New-Frontier«-Mythos, der von Drogen-Gurus wie Timothy Leary oder Acid-Rock-Bands wie Jefferson Airplane und Quicksilver repräsentiert wurde. Das zentrale sinnstiftende Ideal der »New Frontier «, das zu Beginn des Kalten Kriegs aufgefrischt worden war, zeichnete den Green Beret als Nachfahren des Trappers Daniel Boone, als unschuldig-naiven Primitiven, aber technisch versierten Krieger. Präsident Johnson hatte die Mythenpflege grob vernachlässigt. Der Mythos wandte sich gegen das herr- 
schende System. Die US-amerikanische Jugend trat die Flucht in archaischparadiesische Welten an:

»They >dropped out`, growing long hair, wearing buckskin and headbands, sharing peyote-like drugs, and often joining in tribe-like communes. They were taking on the guise of the Indian, the natural man who in the popular culture of their childhoods had been presented as the savage or noble Other confronting the white American on the frontier« (Hellman 1986: 76).

In diesem Klima entstanden vorwiegend kulturfossile irrationale Anschauungen. Der Krieg erstrahlte als metaphysische Größe, als letztmögliches menschliches Abenteuer.

Der Vietnamkrieg, der diese Wandlung des Lebensgefühls erst ermöglichte, lieferte eine geeignete Kulisse für die Inszenierung des kollektiven Deliriums: Vom naiven Sommer-Hit »San Francisco« (1967) von Scott McKenzie mit seichten Textstellen wie »Be sure to wear some flowers in your hair « bis zu den zahllosen Songs über Drogen und Sex von Jimi Hendrix, den Doors, Byrds usw., in denen heute allzu gerne semantische Verweise auf eine Anti-Kriegshaltung gesucht und gefunden werden, obwohl sie in der Regel nichts als den trotzigen Gestus einer egozentrischen, sich unverstanden und im Stich gelassen fühlenden Jugend enthielten. Die ekstatischen Hippie-Tänze von The Grateful Dead standen zwar oberflächlich im Gegensatz zum militärischem Drill, sie indizierten eine intensive Körperlichkeit, archaische Wildheit, sinnliches Jetzt-Erleben - eine scheinbare Autonomie des Individuums. Aber abgesehen davon, dass das Grundmetrum nie verlassen, die betonten Taktteile getanzt wurden, symbolisierte Psychedelic, die Musik der Hippies, eine Freiheit, die nicht politisch begriffen, sondern losgelöst von jeglicher historischer Verantwortung primär im Zusammenhang mit intensiven emotionalen Erlebnissen assoziiert wurde. Nur in wenigen Songs wurde eindeutiger Protest laut und die machtpolitischen Impulse des Krieges enthüllt. Die Urheber und Interpreten der Antikriegs-Songs waren allerdings Musiker, die schon vor Vietnam der amerikanischen Linken angehört hatten, beispielsweise Pete Seeger oder Joan Baez.

In Vietnam waren Pop und Krieg zu einer hemmungslosen Eskapade akkumuliert. Am Ende resümiert der Vietnam-Korrrespondent Michael Herr in seinem Buch Dispatches noch sichtlich benommen von diesem Trip:

»Draußen auf der Straße konnte ich die Vietnamveteranen und die Rock'n' Roll-Veteranen nicht auseinanderhalten. Die Sechziger hatten so viele Verletzte hervorgebracht, ihr Krieg und ihre Musik so lange Energie aus derselben Leitung gezapft, daß sie sich nicht einmal zusammenzutun brauchten. Der Krieg machte sich auf lahme Jahre gefaßt, während der 
Rock'n'Roll gruseliger und gefährlicher als Stierkampf wurde, Rockstars fielen mit einem Mal wie die Leutnants, Rausch und Tod und (natürlich und gewiß) Leben, aber damals sah's nicht so aus. Was ich für zwei Verrücktheiten gehalten hatte, war in Wirklichkeit bloß eine, ich weiß nicht, wie ich klarmachen soll, wie kompliziert das mein Leben machte. Eisig und glühend und wieder in den beschissenen Modder der Kultur runtersteigen: halt durch und mach schön langsam weiter« (Herr 1979: 281).

Auch nach dem Vietnamkrieg wurde die Verbindung Pop und Krieg, das Verschmelzen von Eros und Thanatos als lustvoll erlebt, die Kriegstoten als Statisten eines Schauspiels wahrgenommen oder schlichtweg in den Soundmassakern der Metal-Bands verheizt. Die Band Slayer machte dabei nicht einmal vor den Lagertoren von Auschwitz halt und bettete den Holocaust in ihrem Titel »Angel Of Death « in eine Ästhetik schwarzer Messen, dekadenter Nekrophilie und orgiastischer Gewaltfantasien ein.

Pop und Krieg ästhetisieren sich wechselseitig, stehen in einem symbiotischen Verhältnis zueinander. Krieg liefert Pop nicht nur die Sujets, sondern vor allem eine Schaubühne, auf der sich große Dramen inszenieren lassen. Vom deftigen Schunkelschlager, hektischem Drive der Swingbands, dem Auftrumpfen der Kollektivmacht in den Refrains der Rock- und GospelSongs, über die gekünstelte Vitalität der Disco-, Techno- und Salsa-Beats bis hin zur Koketterie mit dem Grauen: Zugunsten der Huldigung des »carpe diem«-Prinzips haben Pop und Krieg die Grübler, Zweifler und Moralisierer in die Randständigkeit verbannt.

Wenn Pop eine Protesthaltung gegen den Krieg einnimmt, wächst er selten über niveauvollen Kitsch hinaus. Die meisten Versuche münden in ein falsches Pathos, in fatale Höllentrips oder schlichtweg in die Lächerlichkeit. Wenn Pop trauert, dann bleibt er meist in der Sphäre des Sentimentalen und Fatalistischen stecken. Ein aktuelles Beispiel aus dem Mainstream ist der melancholische Ground-Zero-Soundtrack »Only Time« mit dem Motiv der traurigen Schönheit, das den Gestus des Akzeptierens > Ja, so ist es < beinhaltet. Das medial verordnete Rührstück der irischen Sängerin Enya sollte über Wochen »die Betroffenheit der Zuschauer über die Katastrophenbilder vom 11. September ausdrücken « - so hatte jedenfalls die Redaktion der SAT1Nachrichtensendung die nacht entschieden. Nahezu in der gesamten westlichen Welt klangen die abendlichen Sendeblöcke mit diesen visuellakustischen Impressionen vom Ground Zero aus. Die Medien präsentierten das terroristische Attentat als hochästhetische Bildercollage - hier wurde nach allen Regeln filmerischer Inszenierungskunst zu Werke gegangen: Zeitlupe, Überblendtechnik, Einspielung von pixeligen Handkamera-Wackel- 
bildern, die das Publikum spätestens seit Steven Spielbergs Der Soldat James Ryan erwartet.

Irakkrieg, ein Tag vor Kriegsbeginn im Persischen Golf: Vize-Admiral Timothy Keating, Kommandeur der 5. Flotte, betritt eine Bühne auf dem Flugzeugträger USS Constellation. Die kitschige Inszenierung - ein weinender Weißkopfadler mit der Überschrift »We Will Always Remember 9-1101 « als Kulisse, dazu der Queen-Hit »We Will Rock You«, der aus überdimensionalen Lautsprechern dröhnt und Tausende von Soldaten zum Stampfen und Klatschen animiert - beseitigt nicht nur letzte Zweifel, dass die gedemütigte Supermacht Genugtuung am irakischen Menschenmaterial eintreiben will, sondern indiziert: der moderne Krieg wird zusehends als gesamtkunstwerkliches Spektakel rezipiert.

In der Gesellschaft des Spätkapitalismus, die bis in den tiefsten Winkel mit Kulturindustrie durchwirkt ist, die kein Außerhalb mehr zulässt und zu einem Element gewuchert ist, das wir - wie der Kulturwissenschaftler Michael Denning (1991: 267) es ausdrückt - alle »atmen«, scheint eine nachrichtliche Präsentation des Krieges obsolet. »Man könnte denken, daß die langweiligen Filme des Pentagon und von CNN irgendwie zum Opfer von Regisseuren wie Jean-Luc Godard oder denjenigen geworden sind, die Kino mit Video-Überwachung verwechselt haben«, beschrieb 1991 Paul Virilio (1996: 86) seine Eindrücke aus dem ersten Golfkrieg der USA. Der Schriftsteller hatte bereits Mitte der 1980er Jahre in seinem Essay Krieg und Kino auf die Osmose von Kriegs- und Kameratechniken aufmerksam gemacht (vgl. Virilio 1986).

Es scheint, als hätten die Kriegsherren den Regisseur gewechselt und die Inszenierung des Irakkrieges Steven Spielberg überantwortet. Die Bildermaschinen produzierten unentwegt semantische Verweise auf Schlüsselszenen des Zweiten Weltkriegs, den Krieg, der sich aus verständlichen Gründen als gerechter in das Kollektivgedächtnis der Menschheit eingeschrieben hat: Die Stars and Stripes werden von Iwo Jima über Ground Zero zum Paradies-Platz von Bagdad durchgereicht: Dort stülpte ein Soldat der 1. US-Marineinfanteriedivision dem bronzenen Schurken schließlich jenes Fahnentuch über, welches ihm, so versicherte der Krieger, am 11. September im brennenden Pentagon ausgehändigt worden war. Aber viel wichtiger: Wie in Spielbergs Band of Brothers ist die mediale Darstellung des Krieges durch ein unmittelbares Live-Erleben von Action in Echtzeit charakterisiert.

Während die Bilder Nähe suggerieren, schafft Musik Abstand zum Geschehen auf den Schlachtfeldern. Musikhören kann eine Regression bewirken, die bis zur Erfahrung der Spaltung des Raumes reicht. Die Welt, die aus der scheinbaren, >sicheren< Distanz des Draußen geschaut wird, ist 
eine andere. Rainer Schönhammer beschrieb dieses Phänomen am Extrembeispiel des Walkman-Hörens:

»Man ist >draußen<, was man sieht, wird eigentümlich fern, damit aber nicht schlicht negiert, abgeblendet, sondern zum Spektakel, das man in gewisser Weise aufmerksamer betrachtet als ohne die Distanzierung, die ineins aus dem Nicht-Hören der gewohnten Geräuschewelt und der Geborgenheit in der musikalischen Stimmung resultiert. In Befragungen schlägt sich dieses Zum-Spektakel-Werden nicht zuletzt in der Aussage nieder: >Das ist wie Kino« (Schönhammer 1991: 43).

Musik verstärkt die Aura des Krieges. Die Illusion des Krieges wird als angstfreie, lustvolle und berauschende Erfahrung affirmiert. Es verwundert kaum, dass sogar die Kombattanten der High-Tech-Kriege ihr Kriegserlebnis - beispielsweise im Vietnamkrieg oder Golfkrieg - oftmals als Film wahrnehmen. Durch die Einnahme von Drogen, zum Teil von der militärischen Führung verabreicht, und die Dauerbeschallung via Transistorradio - später auch Walkman -, durch die Software Rockmusik entsteht nicht nur der Eindruck eines »schönen« Krieges, sondern ein gefährlicher Taumel zwischen Fiktion und Realität. Michael Herr schildert diese Wahrnehmung folgendermaßen:

»[Es] war, wie esoterischer Musik zu lauschen, du hörtest sie nicht wirklich all die Male, die sie wiederkam, bis dein Atem in sie eingedrungen und ein zusätzliches Instrument geworden war, und dann wars nicht mehr nur Musik, sondern Erfahrung. Leben-als-Film, Krieg-als-(Kriegs-)Film, Krieg-alsLeben« (Herr 1979: 75).

Und Pop liefert die Tonspur für den Krieg als Film-Schauspiel oder das Gesamtkunstwerk Kriegs-Film. Richard Wagners totalitäres Konzept des Gesamtkunstwerks mit seiner extremen Ausrichtung auf Publikumswirksamkeit scheint, wie der Kulturwissenschaftler Moshe Zuckermann (2002: 85ff) ausführt, erst im multimedialen Zeitalter verwirklicht, denn nun können die Sinne optimal stimuliert werden. Die manipulative Massen-Suggestionskraft des Gesamtkunstwerks habe sich, so Zuckermann, im Monumentalismus der Hollywood-»Traumfabrik« erst so richtig entfaltet. Das Gesamtkunstwerk schweißt das Publikum zur Gemeinschaft zusammen. Es hat in besorgniserregender Weise die Entindividualisierung des Menschen beschleunigt und ist schon allein daher pure Ideologie. 


\title{
Literatur
}

Adorno, Theodor W. (1941). »On Popular Music. « In: Zeitschrift für Sozialforschung 9, S. 17-48.

Denning, Michael (1991). "The End of Mass Culture." In: Modernity and Mass Culture. Hg. v. James Naremore und Patrick Brantlinger. Bloomington: Indiana University Press, S. 253-268.

Hellman, John (1986). American Myth and the Legacy of Vietnam. New York: Columbia University Press.

Herr, Michael (1979). An die Hölle verraten. Dispatches. München: Rogner \& Bernhard (5. Aufl.).

Jeckell, Barry A. (2001). "Top Stars >Unite< For D.C. Benefit Concert.«In: Billboard Daily Music News, 9. Oktober, http://www.billboard.com/billboard/daily/ article_display.jsp?vnu_content_id=1075962 (Stand v. 7.5.2004).

Jünger, Ernst (1929). Feuer und Blut. Ein kleiner Ausschnitt aus einer großen Schlacht. Hamburg: Hanseatische Verlagsanstalt.

Kuna, Milan (1993). Musik an der Grenze des Lebens. Musikerinnen und Musiker aus böhmischen Ländern in nationalsozialistischen Konzentrationslagern und Gefängnissen. Frankfurt/M.: Zweitausendeins.

Prox, Lothar (1979). »Melodien aus deutschem Gemüt und Geblüt. "In: Wir tanzen um die Welt. Deutsche Revuefilme 1933-1945. Zusammengestellt v. Helga Belach. München, Wien: Hanser, S. 73-86.

Schönhammer, Rainer (1991). "Walkman-Erfahrungen.« In: Musik als Droge? Zu Theorie und Praxis bewußtseinsverändernder Wirkungen von Musik. Hg. v. Helmut Rösing (= Parlando 1). Mainz: Villa Musica, S. 39-47.

Witte, Karsten (1979). "Gehemmte Schaulust. Momente des deutschen Revuefilms. « In: Wir tanzen um die Welt. Deutsche Revuefilme 1933-1945. Zusammengestellt v. Helga Belach. München, Wien: Hanser, S. 7-52.

Vineyard, Jennifer (2001). »J. Lo, Kid Rock, Ja Rule to Entertain Overseas Troops.« In: MTV News: Archive, 12. März, http://www.mtv.com/news/articles/1451274/ 20011203/kid_rock.jhtml (Stand v. 7.5.2004).

Virilio, Paul (1986). Krieg und Kino. Logistik der Wahrnehmung. München, Wien: Hanser.

Virilio, Paul (1996). Krieg und Fernsehen. München, Wien: Hanser (4. Aufl.).

Zuckermann, Moshe (2002). Kunst und Publikum. Das Kunstwerk im Zeitalter seiner gesellschaftlichen Hintergehbarkeit. Göttingen: Wallstein.

\begin{abstract}
Ever since Woodstock popular music has been considered a culture of freedom and peace. After September 11th, however, a lot of mainstream pop stars supported calls for war and showed an extreme patriotism. Has popular music changed sides? The commitment of pop to peace has always been a lie of the culture industry. There is a genuine link between pop culture and war. It is true that since World War II popular music has not played an important role in manifest war-propaganda but it still serves as soundtrack for a staging of war as Gesamtkunstwerk.
\end{abstract}

